\title{
Uterine infection and immunity in cattle
}

\author{
Iain Martin Sheldon ${ }^{1 *}$, Jennifer C. Price ${ }^{1}$, Matthew L. Turner ${ }^{1}$, \\ John J. Bromfield ${ }^{1,2}$ and James G. Cronin ${ }^{1}$ \\ ${ }^{1}$ Institute of Life Science, College of Medicine, Swansea University, Swansea, SA2 8PP, UK; \\ ${ }^{2}$ Department of Animal Sciences, University of Florida, Gainesville, Florida, USA.
}

\begin{abstract}
Summary
Microbes commonly infect the female genital tract of cattle, causing uterine disease, abortion, and infertility. In particular, postpartum uterine disease develops in about $40 \%$ of dairy cows following infections with Gramnegative Escherichia coli and Gram-positive bacteria, such as Trueperella pyogenes. These infections damage tissues and cause inflammation in the endometrium, and perturb follicle growth and function in the ovary. Innate immunity is the first line of defence against microbes, and inflammatory responses depend on host cellular pattern recognition receptors detecting pathogen-associated molecular patterns. The Toll-like receptor (TLR) family are the most widely studied pattern recognition receptors, with TLR4 binding lipopolysaccharide from Gram-negative bacteria, whilst TLR2 in concert with TLR1 or TLR6 recognise bacterial lipopeptides. Innate immunity in the uterus is not only dependent on immune cells such as neutrophils and macrophages. Endometrial epithelial and stromal cells also express TLRs, and secrete cytokines, chemokines, and prostaglandins in response to lipopolysaccharide and bacterial lipopeptides. In addition, bacterial virulence factors such as pore-forming toxins contribute to the pathology in the endometrium by causing endometrial cytolysis. In the ovary, granulosa cells of antral follicles also possess TLRs and secrete inflammatory mediators in response to pathogen-associated molecular patterns. Furthermore, lipopolysaccharide inappropriately activates the earliest stage of follicle development, and perturbs the normal processes of oocyte maturation and early embryo development. In conclusion, endocrine cells of the bovine endometrium and ovary have roles in innate immunity and the defence against microbes, whilst cellular responses to the pathogen-associated molecular patterns disrupt the physiology of reproduction.
\end{abstract}

\section{Introduction}

Innate immunity is an ancient, evolutionary conserved system across animal phyla. Innate immunity predates the emergence of mammals and the development of viviparity about 340 
million years ago. The last common ancestor of insects and mammals was around 640 million years ago. Yet it was the discovery that the gene Toll was important for immunity in Drosophila melanogaster that reignited the study of innate immunity (Lemaitre, et al. 1996). The discovery in Drosophila and findings of Toll-like receptors in mice was recognised with the award of the Nobel Prize to Jules Hoffmann and Bruce Beutler in 2011 (Lemaitre, et al. 1996, Poltorak, et al. 1998). Formal proof for the importance of innate immunity depends on studies using mutant flies and mice, mice in which genes have been knocked out, transfection of innate immune receptors from cattle and horses into cellular models, and the use of siRNA to knock down gene expression (Ferrandon, et al. 2007, Irvine, et al. 2013, Sauter, et al. 2007, Takeuchi and Akira 2010, Turner, et al. 2014).

Toll is a determinant of dorsoventral patterning of the Drosophila embryo but links between immunity and reproductive biology are tenuous, and silencing components of the innate immune system does not cause overt infertility in mice (Takeuchi and Akira 2010). On the other hand, innate immunity is important for defence of the female genital tract against microbes. Formal evidence for the role of innate immunity in the female reproductive tract has been provided using mutant or knock out mice, or using siRNA in cattle and blocking antibodies in human cells (Elovitz, et al. 2003, Hirata, et al. 2005, Sheldon and Roberts 2010). Microbes commonly cause disease of the genital tract in ruminants when they ascend the cervix or arrive via the peripheral circulation (Sheldon, et al. 2009). These microbes include bacteria, viruses, mycoplasma and parasites, and their diseases include endometritis, embryo mortality, placentitis, abortion, inflammation of the oviduct, and disruption of ovarian function. Amongst ruminants, the incidence and economic impact of infections of the female reproductive tract is highest in dairy cows, then beef cows, followed by sheep and goats. Innate immunity provides the initial rapid response to these microbes and this may be preferable to adaptive immunity because the innate immune system does not recognise the semi-allogeneic embryo in the uterus. Here, we review the concepts underlying the innate immune response, and then discuss the role of innate immunity in the female genital tract with a particular focus on Bos taurus.

\section{Microbes in the female genital tract}

There are a multitude of microbes in the female genital tract with a decreasing gradient from the vagina to the ovary. In all mammals, the vagina is populated by a bacterial microbiome, where Lactobacili are particularly important (Ravel, et al. 2011). The cervix provides a barrier to these bacteria reaching the uterus of normal animals, especially during pregnancy, and the endometrium probably has fewer microbes. However, during and after parturition there is a wide-ranging community of bacteria that can be found in the uterine lumen, as determined by molecular techniques (Machado, et al. 2012). An important remaining question is whether there is a normal microflora in the uterus at times other than during the postpartum period. Although there is uncertainty about the role of each of the microbes found in the uterus after parturition, likely pathogens are Gram-negative Escherichia coli and Gram-positive Trueperella pyogenes, and the anaerobic Fusobacterium, Prevotella and Bacteriodes species. In addition, Brucella abortus, Trueperella pyogenes, Listeria monocytogenes and Salmonella species cause abortion in pregnant cattle. Some microbes associated with infertility, such as Campylobacter fetus and Trichomonas fetus, are adapted for sexual transmission. Pathogens that cause fetal loss include the parasite Neospora caninum, and viruses such as bovine herpesvirus 1 (BoHV1) and bovine viral diarrhoea virus (BVDV). Other recognised pathogens of the female genital tract of cattle include Mycoplasma and Ureaplasma species, and BoHV-4. The ovary rarely acquires an ascending infection with bacteria but viruses, such as BVDV and BoHV-1 infect 
ovarian follicles and even the cumulus-oocyte complex. In most other species, including ruminants, pigs and horses, there are also many similar microbes that cause uterine disease, abortion and infertility.

Many of the specific microbes associated with abortion and infertility in cattle such as Brucella abortus, Campylobacter fetus and Salmonella typhimurium are clearly adapted to cause pathology of the placenta, fetus or endometrium. Amongst the viruses, BoHV-4 is found in many cattle herds but remains latent in macrophages, which is a typical feature of herpesviruses. However, BoHV-4 is associated with postpartum uterine disease and is tropic for the endometrium, particularly stromal cells where the virus causes a rapid cytopathic effect (Donofrio, et al. 2007). One mechanism that may contribute to the association between BoHV-4 and stromal cells is the activation of viral replication by prostaglandin $E_{2}$, which is the predominant prostaglandin secreted by endometrial stromal cells (Donofrio, et al. 2008); although other factors such as TNF $\alpha$ also activate viral replication (Jacca, et al. 2013). These observations may also explain why the virus remains latent in many animals until the onset of bacterial infection of the endometrium after parturition, when secretion of prostaglandin $E_{2}$ and TNF $\alpha$ may activate viral replication and increase the severity of uterine disease. Intriguingly, the viral immediate early protein IE2 transactivates the IL8 gene promoter to attract neutrophils and macrophages, which may increase the availability of cells which the virus can infect and/ or establish latency (Donofrio, et al. 2010).

The most widely investigated disease of the genital tract in cattle is postpartum uterine disease. Genetic selection of Bos taurus dairy cows over the last 60 years has coincided with an increase in the incidence of postpartum uterine disease and a reduction in fecundity. In typical large dairy herds in North America and Europe, up to $40 \%$ of animals have clinical signs of uterine disease and a further $20 \%$ of dairy cows have evidence of subclinical endometritis (Sheldon, et al. 2009). To put this in context, the incidence of postpartum endometritis in other ruminants is usually less than $8 \%$, and veterinary involvement is uncommon, whereas veterinarians spend a considerable amount of their time treating postpartum uterine disease in dairy cattle. Even after successful treatment with antimicrobials or hormones, cattle that had postpartum metritis or endometritis take longer to conceive and are more likely to be infertile. As well as the effects on fertility, uterine infection is associated with lower milk yields particularly if associated with retained placenta (Sheldon, et al. 2004b). The cost of treatment, lost milk production and replacing infertile animals is about $\$ 2$ billion per annum across the dairy industries of North America and Europe (Sheldon, et al. 2009).

The microbes commonly isolated from cases of postpartum endometritis are E. coli, T. pyogenes and Gram-positive anaerobes (Elliot, et al. 1968, Griffin, et al. 1974, Sheldon, et al. 2002, Williams and Sheldon 2003). These microbes are often resistant to multiple antimicrobials (Sheldon, et al. 2004a). Until recently, it was assumed that bacteria that caused uterine disease were non-specific contaminants of the uterus, ascending the genital tract from the vagina, vulva, skin and environment. It now appears that this paradigm needs to be refined because some microbes are adapted to their target environment, or undergo selective enrichment as microbes ascend the tract. When uterine clinical isolates of $E$. coli were examined by multilocus sequence typing, there were specific strains of E. coli, which are distinct from intestinal, mastitis and other extra-intestinal $E$. coli (Sheldon, et al. 2010). These uterine strains of bacteria, called endometrial pathogenic E. coli (EnPEC), were more likely to adhere and invade the epithelial and stromal cells of the endometrium than non-pathogenic strains, and they cause disease when infused into the uterus (Sheldon, et al. 2010).

The severity of endometrial pathology is particularly associated with isolation of Trueperella pyogenes (Bonnett, et al. 1991). This bacterium is often regarded as a commensal organism of cattle but becomes an opportunist pathogen of the uterus after parturition. Recently we 
uncovered the mechanism that underlies this switch, which is associated with a bacterial virulence factor. All clinical isolates of T. pyogenes secrete a cholesterol-dependent cytolysin, called pyolysin (PLO), which causes hemolysis of red blood cells and cytolysis of endometrial cells (Amos, et al. 2014). The family of cholesterol-dependent cytolysins is common to many species of bacteria and is responsible for a wide range of diseases in mammals (Tweten 2005). These toxins cause osmotic cell death when they insert into cholesterol-rich domains in the plasma membrane to form a pore. However, endometrial stromal cells are notably more sensitive to PLO-mediated cytolysis than epithelial cells from the endometrium or hematopoietic immune cells. This differential sensitivity is likely because stromal cells contain more cholesterol than epithelial cells. Indeed, reducing stromal cell cholesterol content using cyclodextrins protects the cells against PLO-mediated cytolysis (Amos, et al. 2014). The marked sensitivity of stromal cells to PLO-mediated cytolysis provides an explanation for how T. pyogenes causes pathology of the endometrium once the protective epithelium is lost after parturition.

There is a considerable inflammatory response following infection of the endometrium. Compared with healthy postpartum cattle, the peripheral plasma of animals with uterine disease has increased concentrations of acute phase proteins such as serum amyloid A, ceruloplasmin and $\alpha-1$ glycoprotein (Sheldon, et al. 2002). Of course, these changes in acute phase proteins or other circulation inflammatory mediators are non-specific, and may be attributed to tissue trauma or infections other than endometritis. However, when endometrium was collected by biopsy from postpartum dairy cows, transcripts for IL1A,IL1B,IL1R and TLR4 were more abundant in the endometrium of diseased than healthy animals in the first week after parturition (Herath, et al. 2009b). (Herath, et al. 2009b). Immuno-reactive protein was detected in the postpartum endometrium for TNF $\alpha$, IL-6, IL-10, IL-1 $\alpha$ and IL-1 $\beta$ (Herath, et al. 2009b). The cytokines were particularly expressed by the glandular epithelium, although IL-6, IL-10 and IL-1 $\alpha$ were evident in the stroma as well. Microarray analysis of endometrium from animals with uterine disease, compared with animals with a normal postpartum period, also found increased abundance of genes such as IL1R, IL8, IL8RB, CXCL5, S100A8, S100A9, and multiple genes encoding complement system proteins (Wathes, et al. 2009). Samples of endometrial cells collected by cytobrush from animals with endometritis also had higher expression of IL1B,TNF, IL8 and CXCL5 than samples from normal animals (Fischer, et al. 2010). Similar observations of expression of inflammatory mediators have been made in the diseased uterus of many species (Sheldon and Bromfield 2011, Sheldon, et al. 2009, Wira and Fahey 2004). Notably, almost all of these inflammatory responses are indicative of innate immunity.

\section{Innate immunity}

Host defence comprises innate immunity and adaptive immunity. Adaptive immunity is dependent on antigen-specific receptors and the response may take several days, depending on whether there has been prior exposure to the antigen. Some microbes that infect the female genital tract such as Brucella abortus generate protective antibody responses, and effective vaccines are available. However, postpartum endometritis often occurs after successive calvings, and adaptive immunity does not appear to be protective. Whereas, innate immunity provides the host with an immediate, non-specific defence system against microbes, which does not depend on prior exposure to microbes.

The innate immune system provides several layers of defence against microbes. The first line of defence is the physical barrier of the epithelium lining the reproductive tract, which ranges from a stratified squamous epithelium in the vagina to the single columnar epithelium of the 
endometrium. The tight junctions between epithelial cells separate the apical and basolateral components of the endometrium, and this barrier counters microbial invasion from the uterine lumen. Furthermore, the mucus layer on the apical surface of epithelia provides an additional obstacle to microbes. The secretion and character of mucus in the genital tract is under the control of ovarian steroids and during oestrus mucus flow increases considerably, whilst the cervical mucus plug provides a physical barrier to ascending infections during gestation. In addition, epithelial cells secrete antimicrobial peptides such as $\beta$-defensins, lingual antimicrobial peptide and tracheal antimicrobial peptide, and glycoproteins such as MUC-1, which all have non-specific effects to counter microbes (Davies, et al. 2008). Antimicrobial peptides are an important component of host defence in the endometrium of several species, being particularly well characterised in the human uterus (Horne, et al. 2008, Sheldon and Bromfield 2011). In addition to the hepatic production of acute phase proteins, epithelial cells in the reproductive tract also express genes for acute phase proteins, although the relative value of localised production during infection remains to be established (Baumann and Gauldie 1994, Chapwanya, et al. 2013). The complement system is also an important part of innate immune defence against microbial infections. This system of about 20 proteins counters microbes by generation of a membranolytic complex at the surface of the pathogen, and by providing opsonins such as $\mathrm{C} 1 \mathrm{~b}$ and $\mathrm{C} 3 \mathrm{~b}$, which interact with cell surface receptors to promote phagocytosis by neutrophils and macrophages. However, to avoid complement actions on normal cells, the latter posses complement regulatory proteins such as CD46, CD55 and CD59 (Gasque 2004). The system of complement proteins is abundant in the female genital tract across species, with studies focussing particularly on rodents, pigs and humans (Harris, et al. 2006, Li, et al. 2002). Complement is regulated by ovarian steroids, and may have roles during fertilization and clearance of surplus spermatazoa in the female genital tract (Harris, et al. 2006, Li, et al. 2002).

Whilst physical barriers and secreted proteins are important for passive defence against microbes in the female genital tract, there are some caveats. First, microbes have evolved counter-measures and strategies to avoid many host defences. For example, some bacteria produce enzymes that lyse mucus to penetrate the protective layer, and many bacteria secrete proteases that can disrupt peptides and proteins produced by the host (Baxt, et al. 2013). Second, pattern recognition receptors, rather than passive defence systems, are principally responsible for initiating rapid inflammatory responses to microbial infections (Takeuchi and Akira 2010).

\section{Pattern recognition receptors}

Much of what drives the innate immune response to microbes depends on cellular pattern recognition receptors. Host cells possess pattern recognition receptors that bind pathogenassociated molecular patterns (PAMPs). These PAMPs are usually highly conserved molecules found in prokaryotes but not eukaryotes, and include lipopeptides, lipopolysaccharides, and microbial RNA and DNA molecules. Mammalian pattern recognition receptors include the TLR family, the cytosolic nucleotide-binding oligomerization domain (NOD)-like receptors (NLRs) and retinoic acid inducible gene (RIG-I)-like receptors (RLRs), and the cell-surface C-type lectin receptors (CLRs) (Blander and Sander 2012, Takeuchi and Akira 2010). Much of the evidence for the importance of TLRs is from studies in mice or using human hematopoietic cells (Takeuchi and Akira 2010). However, studies using transfection of equine or bovine TLRs in cellular models have also provided useful information and difference between species (Irvine, et al. 2013, Werling, et al. 2009) The most widely studied family of innate immune receptors in cattle are the TLRs and there are ten TLRs in cattle (Seabury, et al. 2010). The PAMPs recognised by TLRs are presented in Table 1. 
Table 1 Pattern recognition receptors, their localization, and the principal pathogen-associated molecular patterns that they bind

\begin{tabular}{lll}
\hline Receptor & Localization & Pathogen-associated molecular patterns \\
\hline TLR1 & Plasma Membrane & Bacterial lipopeptides \\
TLR2 & Plasma Membrane & Bacterial lipopeptides, glycolipids \\
TLR3 & Endosome & Viral double-stranded RNA \\
TLR4 & Plasma Membrane & Lipopolysaccharide \\
TLR5 & Plasma Membrane & Flagellin \\
TLR6 & Plasma Membrane & Bacterial lipopeptides \\
TLR7 & Endosome & Microbial single-stranded RNA \\
TLR8 & Endosome & Microbial single-stranded RNA \\
TLR9 & Endosome & CpG-rich bacterial and viral DNA \\
TLR10 & Plasma Membrane & Unknown \\
RIG-I & Cytoplasm & Short double stranded RNA \\
NOD1 & Cytoplasm & Peptidoglycan \\
NOD2 & Cytoplasm & Peptidoglycan \\
\hline
\end{tabular}

The TLRs that span the plasma membrane of cells (Fig. 1) have an extracellular component containing leucine-rich repeats, a trans-membrane domain, and an intracellular Toll/interleukin-1 receptor (TIR) domain; this TIR domain is homologous to the intracellular domain of the IL-1 receptor family. The leucine-rich repeats are also important for ligand binding in the intracellular TLRs. The co-receptors MD-2 and CD14 are important for LPS binding to TLR4, whilst CD14 and CD36 augment TLR2 function but are not essential for signalling. There is some species variation in TLR ligand specificity, and formal testing of the role of each TLR needs further work. For example, based on gene silencing experiments using short interfering RNA (siRNA), whilst TLR1 binds triacylated lipopeptides when it heterodimerizes with TLR2, bovine TLR6 is less discriminatory than in mice or humans and binds not only diacylated but also triacylated lipopeptides (Turner, et al. 2014, Werling, et al. 2009). Transfection of bovine TLRs into human embryonic kidney cells has also been used to explore the responses to ligands (Sauter, et al. 2007). In general the TLRs that sense bacteria are in the plasma membrane (TLR1, TLR2, TLR4, TLR5 and TLR6), whilst the remainder pattern recognition receptors are intracellular, and are principally used to detect microbes that invade cells (Table 1).

Activation of TLRs with bacterial PAMPs typically leads to production of the cytokines IL-1, IL-6 and TNF $\alpha$, and chemokines such as IL-8. On the other hand, viral PAMPs often stimulate the release of interferons. The inflammatory cytokines bind their cognate receptors leading to increased permeability of vasculature, and secretion of antimicrobial peptides, eicosanoids and reactive oxygen species. Distant to the site of infection, cytokines in the peripheral plasma drive systemic inflammatory responses; these include pyrexia, generalised vasodilation, and release of acute phase proteins from hepatocytes. Chemokines attract and activate neutrophils and monocytes to the site of infection. Trauma to tissues associated with cell destruction by microbes or by physical damage during parturition, may contribute to inflammation in the endometrium. The ability of pattern recognition receptors to bind damage-associated molecular patterns (DAMPs) may provide an explanation linking tissue trauma to inflammation (Chen and Nunez 2010). Mammalian 


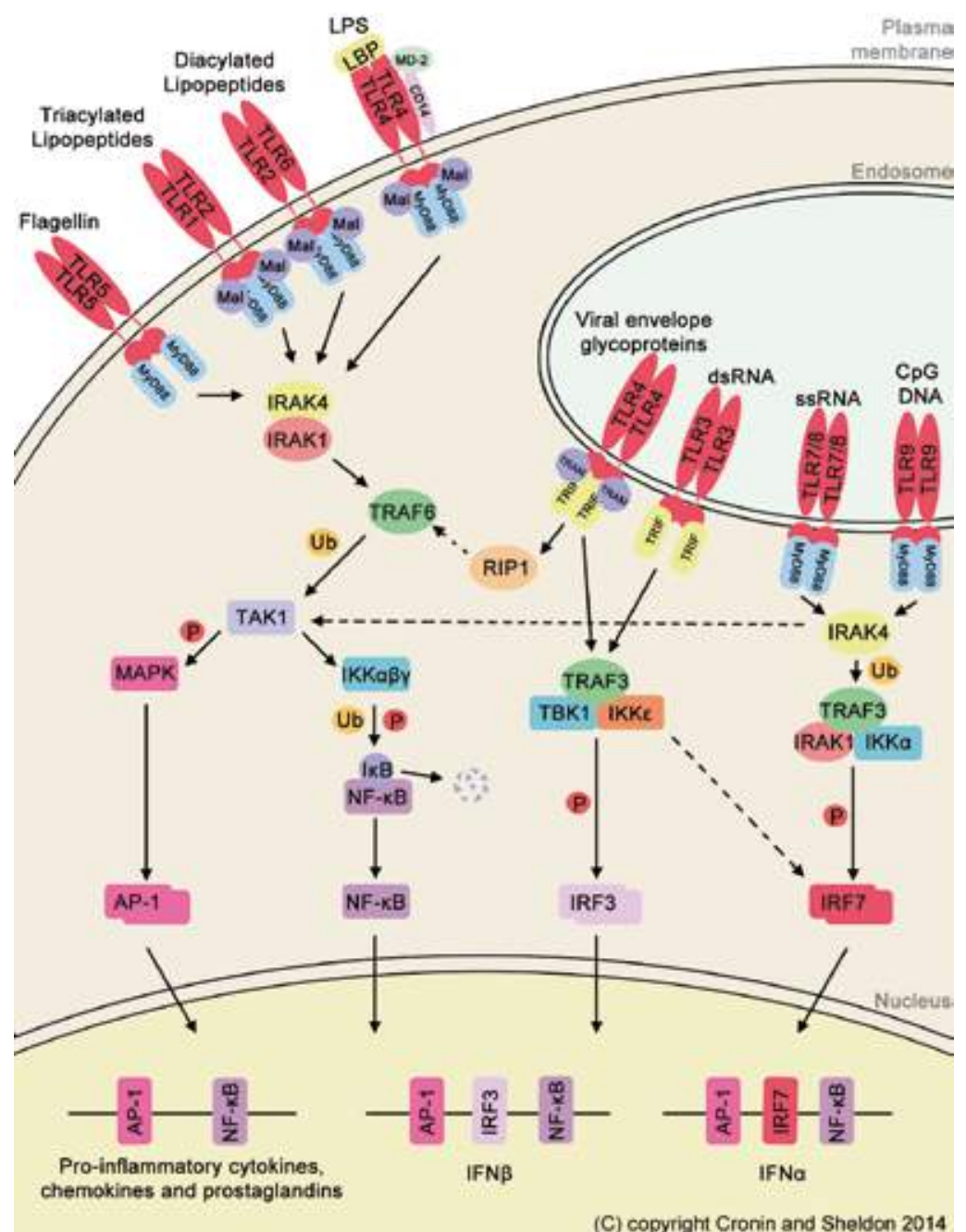

\section{Fig. 1 Signalling pathways of the Toll-like receptors}

During infection PAMPs initiate signalling pathways by interacting with TLRs, resulting in formation of homoor heterodimers and activation of cytosolic Toll/IL-1 receptor (TIR) domains. Downstream signalling pathways are initiated through four adaptor proteins: MyD88; Mal (also known as TIRAP), TRIF, or TRAM. All TLRs, with the exception of TLR3, use the MyD88-dependent pathway. Mal acts as a bridge to recruit MyD88 to TLR2 and TLR4 signalling. The adaptor TRIF is used in TLR3 signalling and, in association with TRAM, in endosomal TLR4 signalling. In the MyD88-dependent pathway, MyD88 associates with IRAK4, IRAK1 (and/or IRAK2; not illustrated). IRAK4 in turn phosphorylates IRAK1 and promotes association with TRAF6, which serves as a platform to recruit and activate the kinase TAK1. Activated TAK1 leads to the activation of the MAPK cascade. The MAPKs ERK, JNK and p38 enhance the expression of pro-inflammatory cytokines via activation of AP-1 (for simplicity, only activation of AP-1 is illustrated in the diagram). TAK1 also leads to the activation the IKK complex, composed of IKK $\alpha$, IKK $\beta$, and IKK $\gamma$ (also known as NEMO), which results in the phosphorylation and ubiquitination of $I \kappa B$. Ubiquitination results in degradation of $I \kappa B$, freeing NF- $\kappa B$ to translocate from the cytoplasm to the nucleus, where it activates gene expression in concert with AP-1. Endosomal TLR3 and TLR4 signalling, through the adaptor protein TRIF, leads to activation of TBK1 or IKKE, inducing IRF3 dimerization and translocation into the nucleus where it induces transcription of type I IFN and IFN-inducible genes, including IFN $\beta$. Downstream of TLR 7, 8, and 9, IRF7 is directly phosphorylated by IRAK1 and translocates into the nucleus to induce the expression of type I IFN and IFN-inducible genes, including IFN $\alpha$. 
DAMPs include HMGB1, IL-1 $\alpha$, mitochondrial DNA and ATP, which are released from dying or damaged cells (Chen and Nunez 2010). However, in the scaling of the inflammatory response, DAMPs are perceived as less of a threat than PAMPs (Blander and Sander 2012).

The innate immune response to PAMPs and DAMPs is most commonly associated with macrophages, neutrophils, and dendritic cells. However, a range of cells other than hematopoietic immune cells also expresses pattern recognition receptors. For example, epithelial cells and fibroblastic stromal cells express functional TLRs in the bovine endometrium, mammary gland and intestine. So, the overall inflammatory response to pathogens depends on the sum of the actions of multiple cell types rather than just specialized immune cells. One obvious question is why host cellular responses to pathogens are greater than when the same cells encounter commensal microbes? Many of the PAMPs are found in both pathogens and commensals, but PAMPs form only one component of the virulence factors possessed by pathogens and the activity of these microbes differs markedly from commensals. The impact of pathogens on the host is enhanced by pore-forming toxins, bacterial secretion systems, and bacterial mRNA (Blander and Sander 2012). Furthermore, unlike commensal organisms, pathogens tend not only to colonise tissues but also to invade the cells of the female genital tract and cause cell death (Donofrio, et al. 2007, Sheldon, et al. 2010). So, the scale of the inflammatory response to microbes is dependent on multiple factors including microbial virulence effectors, host tissue factors, and on the regulation of intracellular signalling pathways associated with innate immunity.

\section{TLR signalling pathways}

Based on the TIR domain-containing adaptor molecules recruited to TLRs, signalling can be divided into two distinct pathways (Fig. 1). With the exception of TLR3, MyD88 is essential for downstream signalling of all TLRs (Moresco, et al. 2011, Takeuchi and Akira 2010). The MyD88dependent signalling pathways induce phosphorylation of MAPK, and the canonical IKK complex (Fig. 1). The MAPK cascade (JNK, ERK1/2 and p38) then activates AP-1, a transcription factor complex; whereas, activation of the IKK complex results in phosphorylation and degradation of $I \kappa B$, which allows the transcription factor NF- $\kappa B$ to translocate into the nucleus. Typical genes transcribed by activation of these signalling pathways include cytokines (IL1A, IL1B, IL6 and TNFA), chemokines (IL8 and CXCL1), and lipid inflammatory mediators (PLA2, PGHS and PGES) (Fig. 1). The MyD88-independent pathways not only lead to activation of MAPK and NF- $\kappa B$ signalling, but also phosphorylation of IRF3 and IRF7, which translocate into the nucleus and induce expression of type I interferons (IFN; Fig 1). The type I IFNs orchestrate diverse immune responses to infection. Although typically considered antiviral cytokines, type I IFNs are also induced by bacterial pathogens (Karaghiosoff, et al. 2003). Evasion of the innate immune response is important for viral persistence (Goubau, et al. 2013). Indeed, early infection of the bovine foetus with noncytopathic BVDV in utero does not induce a type I IFN response and can lead to viral persistence for the life of that animal. Furthermore, infection with noncytopathic BVDV inhibits the synthesis of IFN $\alpha$ and IFN $\beta$ in response to the TLR9 ligand poly(IC) or the TLR3 ligand synthetic double-stranded RNA (Schweizer and Peterhans 2001).

The DAMP IL-1 $\alpha$ and pro-inflammatory cytokine IL-1 $\beta$ both bind to the IL-1 receptor type 1 (IL-1R1), which also leads to the activation of NF-אB and MAPKs and in some case IFN regulatory factors (IRFs). Interleukin- $1 \beta$ is an important pro-inflammatory mediator and its activity is tightly controlled by expression, maturation and secretion (Garlanda, et al. 2013). Interleukin-1 $\beta$ is synthesized as an inactive precursor (pro-IL-1 $\beta$ ) in response to PAMPs. Maturation of pro-IL-1 $\beta$ is dependent on the intracellular NOD-like receptors (NLRs) and their formation into multiprotein 
complexes called inflammasomes (Schroder and Tschopp 2010). The most fully characterised inflammasome involved in pro-IL-1 $\beta$ maturation is the NOD-, LRR-and pyrin domain-containing 3 (NLRP3; also known as NALP3 or cryopyrin) inflammasome. This multiprotein complex consists of the NLRP3 scaffold, the ASC adaptor and caspase-1. Clustering of pro-caspase 1 on oligomerized NLRP3 results in auto-activation of caspase 1-dependent processing of pro-IL-1 $\beta$ to mature IL-1 $\beta$ (Agostini, et al. 2004).

\section{Innate immunity in the endometrium}

Endometrium from healthy animals express gene transcripts for all the pattern recognition receptors examined, including the TLRs (Davies, et al. 2008). Furthermore, endometrial biopsies collected from healthy and diseased postpartum dairy cows expressed mRNA for all ten TLRs (Herath, et al. 2009b). At the protein level, TLR4 protein was also confirmed in the bovine and murine endometrium using immunohistochemistry (Herath, et al. 2009b, Sheldon, et al. 2010). Ex vivo organ cultures of intact endometrium collected from beef or dairy cattle accumulate IL-1 $\beta$, IL-6 and IL-8 protein, and prostaglandin $\mathrm{E}_{2}$ when challenged with live clinical isolates of $E$. coli or $T$. pyogenes (Borges, et al. 2012). Similarly, chopped tissue explants of bovine endometrium collected from healthy animals and treated with LPS also accumulate prostaglandin $E_{2}$ and prostaglandin $F_{2}$ (Herath, et al. 2006). Taken together, these data support the concept that endometrial tissues are able to mount a rapid and robust inflammatory response indicative of innate immunity. However, healthy endometrium is comprised of multiple cell types including epithelium, stroma, endothelium and professional immune cells, whilst there is an influx of monocytes and neutrophils into infected endometrium. The step forward we made was to explore whether the epithelial and stromal cells might have roles in innate immunity, in addition to the professional immune cells.

Pure populations of endometrial epithelial and stromal cells expressed a range of TLRs (Davies, et al. 2008). Furthermore, epithelial and stromal cells, as well as macrophages, not only expressed TLR4 but also mRNA for the co-receptors MD2 and CD14, so all the machinery necessary to respond to LPS was present (Herath, et al. 2006). Initial studies focussed on TLR4 because $E$. coli is a common isolate of the uterine lumen after parturition, and LPS is detectable in the uterine lumen of diseased animals (Williams, et al. 2007). Addition of LPS stimulates a concentration dependent accumulation of prostaglandin $\mathrm{F}_{2 \alpha}$ and prostaglandin $\mathrm{E}_{2}$, and IL-6, CXCL1, CXCL2, CXCL3 and IL-8 protein in the supernatants of epithelial or stromal cells (Cronin, et al. 2012, Turner, et al. 2014). Similarly, endometrial epithelial and stromal cells collected from wild type, but not TLR4 knockout mice, responded to LPS by secreting IL-6, CXCL1, CCL20 and prostaglandin $\mathrm{E}_{2}$ (Sheldon and Roberts 2010). These individual cellular responses to PAMPs mirror the inflammatory responses to PAMPs or bacteria by bovine cells in situ, studied in vivo or using ex vivo organ cultures (Borges, et al. 2012, Herath, et al. 2009b, Wathes, et al. 2009) In addition, LPS increased the phosphorylation of MAPK and nuclear translocation of NFKB by endometrial cells (Cronin, et al. 2012). Similar inflammatory mediator responses, including the secretion of IL-6 and IL-8 protein, are evident from endometrial epithelial and stromal cells treated with ligands that bind TLR2/TLR1 and TLR2/TLR6 heterodimers (Turner, et al. 2014). However, whilst there was increased mRNA expression for IL1B, appreciable amounts of mature IL-1 $\beta$ protein are rarely detected; presumably this reflects a lack of activation of the inflammasome by ultrapure PAMPs engaging surface TLRs. On the other hand, live E. coli or T. pyogenes stimulate multiple TLRs and NLRs, leading to the secretion of IL-1 $\beta$ protein (Amos, et al. 2014, Borges, et al. 2012). Responses to flagellin were also not evident using highly purified, endotoxin-free preparations; although, earlier commercial preparations of flagellin did appear to stimulate cellular 
responses. Similar experiences with early preparations of some PAMPs and DAMPs have caused some confusion, and great care should be taken when evaluating data in the literature. Indeed, to confirm cellular responses to PAMPs are specific, it is important to use siRNA to silence target genes. Silencing TLR1, TLR2, TLR4 and TLR6 gene expression in bovine endometrial cells using siRNA significantly reduces cellular responses to their cognate PAMPs (Fig. 2). Similarly, uterine inflammatory responses can be blunted using genetic mutations in mice, knockout mice, or using blocking antibodies with human cell (Elovitz, et al. 2003, Hirata, et al. 2005, Sheldon and Roberts 2010). Many of these TLR pathways also have feedbacks that regulate the inflammatory response, and in the bovine endometrium STAT and SOCS molecules modulate the secretion of IL-6 and the chemokine IL-8 (Cronin, et al. 2012). Taken together these data support the concept that the epithelial and stromal cells in the endometrium have roles in the initial sensing and response to pathogens. Whilst a link between inflammation in the endometrium and infertility is intuitive, it was less obvious whether these infections might impact ovarian function.

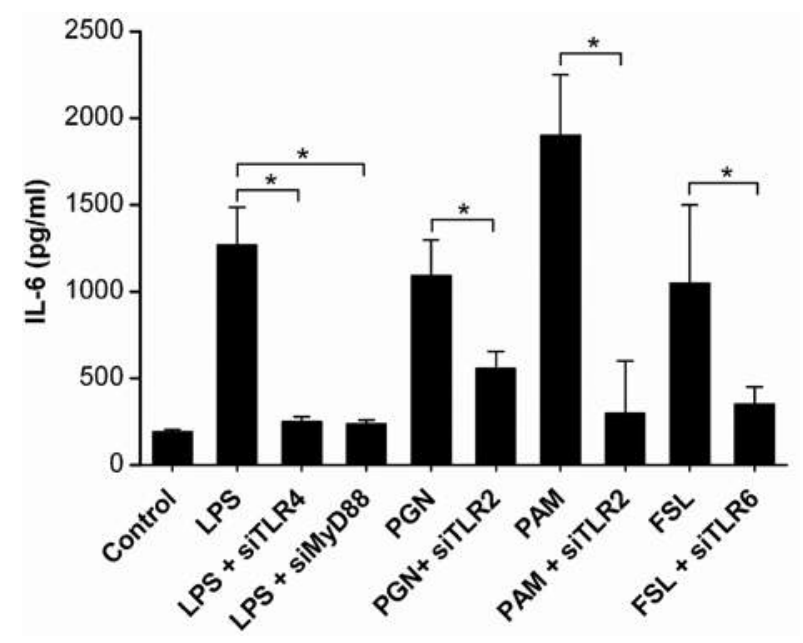

Fig. 2 Silencing TLR expression reduces endometrial cell responses to PAMPs. Control medium or medium containing LPS, peptidoglycan (PGN), a triacylated lipopeptide (PAM) or a diacylated lipopeptide (FSL-1) was applied to normal bovine endometrial stromal cells or cells in which TLR4 and MyD88, TLR2, TLR1 or TLR6, respectively, had been silenced by siRNA. Supernatants were collected and IL- 6 measured by ELISA. Data are presented as mean + SEM from 4 independent experiments, and analyzed by ANOVA with Bonferroni post hoc test, $* \mathrm{P}<0.05$.

\section{Innate immunity in the ovary}

For many years the principal thinking around the relationship between the components of the female reproductive system during reproductive cycles has been that the ovary regulates uterine function. Indeed, oestradiol and progesterone from ovarian follicles and the corpus luteum, respectively, are essential for the physiological function of the endometrium and establishment of pregnancy. However, the relationship between the ovary and uterus is not unidirectional and prostaglandins released by the endometrium regulate corpus luteum function, mainly by localized vascular pathways that provide a functional connection between the two organs. After parturition and before an animal is likely to conceive there must be uterine involution, elimination of bacterial contamination of the uterus, regeneration of the endometrium, and return of ovarian cyclic activity. Surprisingly, ovarian function appears to have little effect on uterine involution and elimination of bacterial contamination of the uterus. Indeed, multiple efforts to use oestradiol 
or other hormones to hasten the rate of uterine involution have not been successful (Sheldon, et al. 2003a, Sheldon, et al. 2003b). Conversely, for the standard animal model of endometritis in cattle, exogenous progesterone increases the severity and duration of purulent discharge following scarification of the endometrium and infusion of T. pyogenes (Ayliffe and Noakes 1982, Rowson, et al. 1953). However, when there is uterine infection, exogenous oestradiol or induction of oestrus using exogenous prostaglandin $\mathrm{F}_{2 \alpha}$ when there is a corpus luteum in the ovary, are reasonably effective treatments for resolving the clinical signs of endometritis. This may be in part because endometritis is associated with increased prostaglandin $\mathrm{E}_{2}$ production, and reduced prostaglandin $\mathrm{F}_{2 \alpha}$ (Herath, et al. 2009a). Notably, LPS stimulated increased prostaglandin $\mathrm{E}_{2}$ production by increased abundance of phospholipase A2 group 6 protein in bovine endometrial cells.

Whilst testing whether oestradiol might enhance the rate of uterine involution or improve endometrial health, we were surprised to find the opposite was more likely. Dairy cows with postpartum uterine infections have a slower growth of the dominant follicle, lower peripheral plasma oestradiol concentrations, and were less likely to ovulate (Sheldon, et al. 2002). One possibility is that the inflammatory mediators from the uterus may reach the ovary via the localised counter-current mechanism, as used by prostaglandin $\mathrm{F}_{2 \alpha}$ during luteolysis. Indeed, IL-6 and TNF $\alpha$ perturb bovine ovarian follicular cell steroidogenesis (Alpizar and Spicer 1994, Spicer 1998). These mechanisms are likely to be applicable across species as cytokines also modulate the production of ovarian steroids in rodents and in humans (Rice, et al. 1998, Roby and Terranova 1990). We also considered whether PAMPs might play a role in perturbing ovarian function because the concentrations of LPS in follicular fluid aspirated from dominant follicles are increased in line with the severity of uterine disease, whilst absent in animals with a normal endometrium (Herath, et al. 2007). However, healthy ovarian follicles do not appear to contain hematopoietic immune cells, as determined by the absence of CD45 or MHCIl expression (Bromfield and Sheldon 2011, Herath, et al. 2007). Addition of LPS to bovine granulosa cells in vitro reduced oestradiol production, although there was no effect on androstenedione production by theca cells (Herath, et al. 2007). The mechanism is a reduction in aromatase at the gene and the protein level in granulosa cells treated with LPS (Herath, et al. 2007, Price, et al. 2013). Interestingly, granulosa cells isolated from growing or dominant ovarian follicles express most of the TLRs (Bromfield and Sheldon 2011, Price, et al. 2013). Furthermore, PAMPs such as bacterial LPS or bacterial lipopeptides stimulate an inflammatory response by granulosa cells, with the secretion of IL-1 $\beta$, IL-6, CXCL1, CXCL2, CXCL3 and IL-8 protein (Bromfield and Sheldon 2011, Price, et al. 2013). Granulosa cells responded rapidly to LPS or lipopeptides with rapid phosphorylation of p38 and ERK, and inhibiting intracellular MAPK signalling pathways reduced the inflammatory response to their cognate PAMPs (Bromfield and Sheldon 2011, Price, et al. 2013). In addition, silencing TLR4 or TLR2 in bovine granulosa cells using siRNA, reduced the secretion of IL-6 in response to their cognate PAMPs (Bromfield and Sheldon 2011, Price, et al. 2013). The secretion of IL-6 protein in response to PAMPs by isolated granulosa cells reflects the increased IL- 6 secretion by cells in situ in cultured ovarian follicles (Bromfield and Sheldon 2013). Innate immune receptors also appear to be important for sensing and responding to PAMPs in rodents, humans and chickens (Richards, et al. 2008, Sheldon and Bromfield 2011, Woods, et al. 2009, Yan, et al. 2013). Taken together these data support our concept that granulosa cells within ovarian follicles have roles in innate immunity. To take this a step further we then investigated whether PAMPs might impact earlier stages of follicle development or the final stages of cumulus-oocyte expansion.

Follicle development from the primordial stage to ovulation takes 120 to 150 days in cattle. Bovine ovarian cortex was cultured ex vivo and LPS reduced the primordial follicle pool, with an associated increase in primordial follicle activation (Bromfield and Sheldon 2013). Furthermore, LPS treatment modulated key intracellular regulators of follicle activation with loss of the primordial 
follicle PTEN and cytoplasmic translocation of FOXO3. In addition, the LPS-treated ovarian cortex culture supernatants accumulated IL-1 $\beta$, IL-6, and IL-8 protein in a concentration-dependent manner. A negative impact of LPS on primordial follicle development in vivo was also identified using wild type but not TLR4 knockout mice (Bromfield and Sheldon 2013). Considering the long development period of ovarian follicles from the primordial stage to ovulation, these observations may provide a mechanism for long-term effects of uterine disease on fertility. However, not all stages of follicle development are equally sensitive to PAMPs, and LPS did not affect the growth and viability of individually cultured secondary follicles or their enclosed oocytes (Bromfield and Sheldon 2013).

Considering the later stages of ovarian follicle development, LPS stimulated IL-6 secretion from cumulus-oocyte complexes and stimulated expansion in vitro (Bromfield and Sheldon 2011); this may contribute to infertility because expansion is normally closely coordinated with ovulation. This work is similar to mice where cumulus-oocyte complexes exposed to bacterial lipopolysaccharide exhibit enhanced phosphorylation of MAPK and NF-אB, and increased expression of IL6 (Shimada, et al. 2006). We also reasoned that if cumulus granulosa cells respond to LPS and secrete IL-6, LPS might perturb the oocyte via the cytoplasmic trans-zonal projections from granulosa cells that synapse on the oolema. Indeed, high concentrations of LPS increased rates of meiotic arrest

A CoC differentially expressed genes
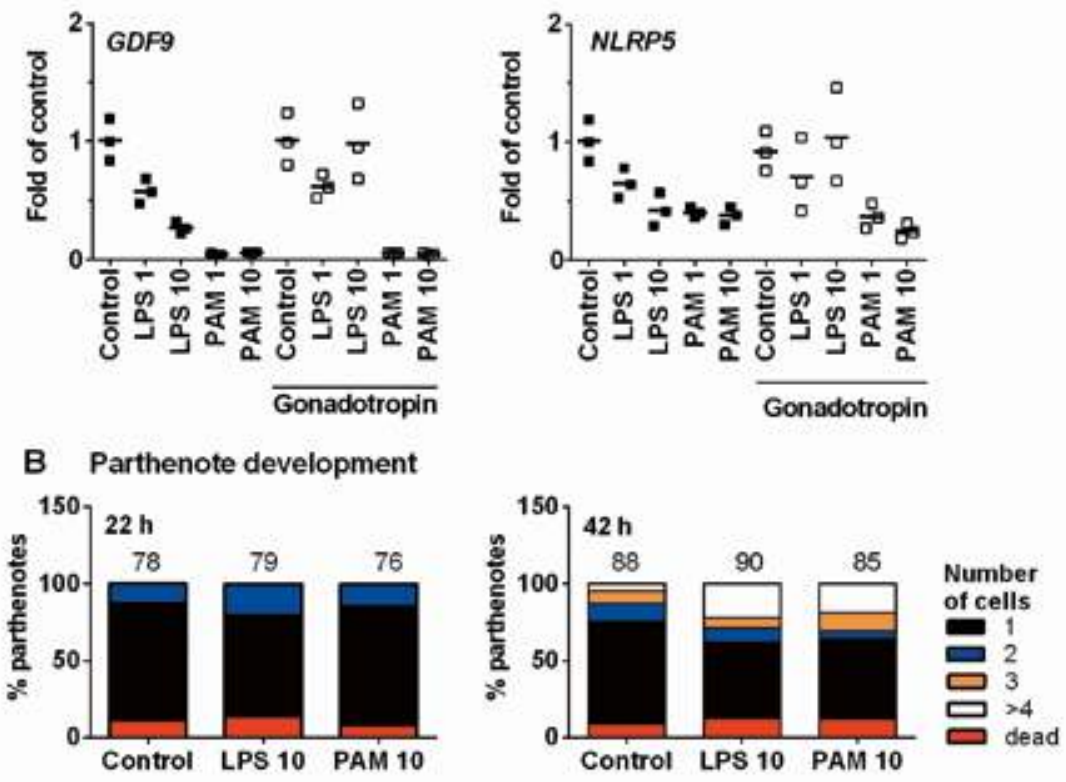

Fig. 3 Early parthenogenic embryo development and innate immunity. Bovine cumulus-oocyte complexes (COCs) were matured in control medium or medium containing $2.5 \mu \mathrm{g} / \mathrm{ml} \mathrm{FSH}$ and $10 \mu \mathrm{g} / \mathrm{ml} \mathrm{LH}$ in the presence or absence of 1 or $10 \mu \mathrm{g} / \mathrm{ml}$ LPS or PAM. (A) After $3 \mathrm{~h}$, expression of genes associated with oocyte maturation (NLRP5, GDF9) was estimated by qPCR, normalised to L19 gene expression. Data expressed relative to control from 3 independent experiments (total 915 COCs), and presented as dot plots with horizontal line representing the mean. (B) After $24 \mathrm{~h}$, to stimulate parthogenesis, the cumulus was stripped from the oocytes using hyaluronidase, and oocytes were cultured in control medium containing $5 \mu \mathrm{M}$ ionomycin for $4 \mathrm{~min}$, followed by $2 \mathrm{mM}$ 6-dimethylaminopyridine for $4 \mathrm{~h}$. The oocytes were then incubated in embryo culture medium, and at $22 \mathrm{~h}$ and $42 \mathrm{~h}$ pathogenic progression was evaluated using phase contrast microscopy by counting the number of cells. The experiment was repeated on 5 independent occasions, and the number of parthenotes is indicated above each bar. 
and germinal vesicle breakdown failure (Bromfield and Sheldon 2011). In addition, treatment of cumulus-oocyte complexes with LPS or PAM perturbed expression of genes involved in oocyte maturation (Fig. 3; P < 0.05), although further work is needed to confirm these effects at the protein level. Furthermore, oocytes which were in vitro maturated in the presence of LPS tended towards increased parthenogenic cleavage rate after $22 \mathrm{~h}(\mathrm{P}<0.06)$, and there was a significantly increased parthenogenic cleavage rate after $42 \mathrm{~h}$ in the presence of LPS or PAM (Fig. 3; P < 0.01). Whilst these data are relevant to uterine disease, other diseases such as mastitis might also act on the ovary in a similar manner (Asaf, et al. 2014, Lavon, et al. 2011).

\section{Conclusion}

Microbial infections of the mammalian female genital tract are common and cause infertility, and this is particularly important in cattle. Innate immunity is the first line of defence against invading microbes, leading to inflammation and helping to orchestrate the adaptive immune response across species. Innate immunity is central to the early defence against microbes in the uterus. Endometrial epithelial and stromal cells, as well as immune cells, detect bacteria via TLR-dependent mechanisms and generate inflammatory responses with secretion of cytokines, chemokines and prostaglandins (Fig. 4). Ovarian function is also perturbed by pathogen associated molecular patterns, which are recognized by TLRs on the granulosa cells.

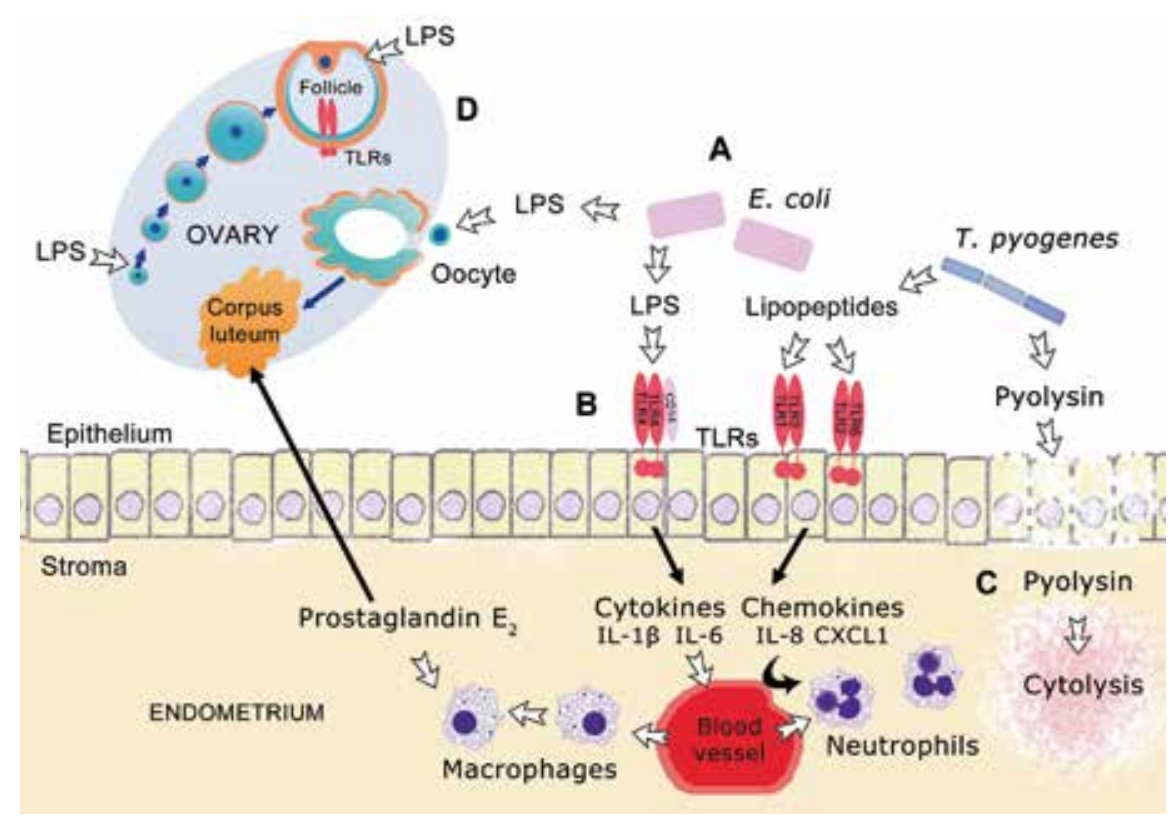

Fig. 4 Innate immunity and reproduction

(A) Gram-negative E. coli and Gram-positive T. pyogenes infect the endometrium. Endometrial epithelial and stromal cells detect bacterial PAMPs such as LPS and lipopeptides, which bind to TLRs. (B) Activation of TLRs leads to the secretion of cytokines, chemokines and prostaglandins, which attract and activate macrophages and neutrophils. (C) Pyolysin secreted by T. pyogenes also causes damage to the endometrium, particularly by causing stromal cell lysis. (D) Inflammatory mediators such as prostaglandin $\mathrm{E}_{2}$ and PAMPS such as LPS, also impact ovarian function, from primordial follicle development, through to follicle growth and function, and even oocyte health and the lifespan of the corpus luteum. 


\section{Acknowledgements}

The work in the Sheldon laboratory is funded by the Biotechnology and Biological Sciences Research Council (BBSRC; Grant numbers BB/I017240/1 and BB/006592/1) in the UK, Dr ML Turner held a BBSRC Doctoral Training Studentship (BB/F017596/1), and Dr JC Price held a BBSRC CASE PhD Studentship (BB/G017131/1).

\section{References}

Agostini, L, F Martinon, K Burns, MF McDermott, PN Hawkins, and J Tschopp 2004 NALP3 forms an IL1 beta-processing inflammasome with increased activity in Muckle-Wells autoinflammatory disorder. Immunity 20 319-325.

Alpizar, E, and LJ Spicer 1994 Effects of interleukin-6 on proliferation and follicle-stimulating hormone-induced estradiol production by bovine granulosa cells in vitro: dependence on size of follicle. Biology of Reproduction 49 38-43.

Amos, MR, GD Healey, RJ Goldstone, S Mahan, A Duvel, HJ Schuberth, O Sandra, P Zieger, I DieuzyLabaye, DGE Smith, and IM Sheldon 2014 Differential endometrial cell sensitivity to a cholesterol-dependent cytolysin links Trueperella pyogenes to uterine disease in cattle Biology of Reproduction 90 54,51-13.

Asaf, S, G Leitner, O Furman, Y Lavon, D Kalo, D Wolfenson, and Z Roth 2014 Effects of Escherichia coli- and Staphylococcus aureus-induced mastitis in lactating cows on oocyte developmental competence. Reproduction 147 33-43.

Ayliffe, TR, and DE Noakes 1982 Effects of exogenous oestrogen and experimentally induced endometritis on absorption of sodium benzylpenicillin from the cow's uterus. Veterinary Record 110 96-98.

Baumann, H, and J Gauldie 1994 The acute phase response. Immunology Today 15 74-80.

Baxt, LA, AC Garza-Mayers, and MB Goldberg 2013 Bacterial subversion of host innate immune pathways. Science 340 697-701.

Blander, JM, and LE Sander 2012 Beyond pattern recognition: five immune checkpoints for scaling the microbial threat. Nature Reviews. Immunology 12 215-225.

Bonnett, BN, SW Martin, VP Gannon, RB Miller, and WG Etherington 1991 Endometrial biopsy in HolsteinFriesian dairy cows. III. Bacteriological analysis and correlations with histological findings. Canadian Journal of Veterinary Research 55 168-173.

Borges, AM, GD Healey, and IM Sheldon 2012 Explants of intact endometrium to model bovine innate immunity and inflammation ex vivo. American Journal of Reproductive Immunology 67 526-539.

Bromfield, JJ, and IM Sheldon 2011 Lipopolysaccharide initiates inflammation in bovine granulosa cells via the TLR4 pathway and perturbs oocyte meiotic progression in vitro. Endocrinology 152 5029-5040.

Bromfield, JJ, and IM Sheldon 2013 Lipopolysaccharide reduces the primordial follicle pool in the bovine ovarian cortex ex vivo and in the murine ovary in vivo. Biology of Reproduction $\mathbf{8 8} 98$.

Chapwanya, A, KG Meade, ML Doherty, JJ Callanan, and C O'Farrelly 2013 Endometrial epithelial cells are potent producers of tracheal antimicrobial peptide and serum amyloid A3 gene expression in response to E. coli stimulation. Veterinary Immunology and Immunopathology 151 157-162.

Chen, GY, and G Nunez 2010 Sterile inflammation: sensing and reacting to damage. Nature Reviews. Immunology 10 826-837.

Cronin, JG, ML Turner, L Goetze, CE Bryant, and IM Sheldon 2012 Toll-Like Receptor 4 and MYD88Dependent Signaling Mechanisms of the Innate Immune System Are Essential for the Response to Lipopolysaccharide by Epithelial and Stromal Cells of the Bovine Endometrium. Biology of Reproduction 86 51, 51-59.

Davies, D, KG Meade, S Herath, PD Eckersall, D Gonzalez, JO White, RS Conlan, C O'Farrelly, and IM Sheldon 2008 Toll-like receptor and antimicrobial peptide expression in the bovine endometrium. Reproductive Biology and Endocrinology 653.

Donofrio, G, A Capocefalo, V Franceschi, S Price, S Cavirani, and M Sheldon 2010 The chemokine IL8 Is up-regulated in bovine endometrial stromal cells by the BoHV-4 IE2 gene product, ORF50/Rta: A step ahead toward a mechanism for BoHV-4 induced endometritis. Biology of Reproduction 83 919-928.

Donofrio, G, S Herath, C Sartori, S Cavirani, CF Flammini, and IM Sheldon 2007 Bovine herpesvirus 4 (BoHV-4) is tropic for bovine endometrial cells and modulates endocrine function. Reproduction 134 183-197.

Donofrio, G, L Ravaneti, S Cavirani, S Herath, A Capocefalo, and IM Sheldon 2008 Bacterial infection of endometrial stromal cells influences bovine herpesvirus 4 immediate early gene activation: a new insight into bacterial and viral interaction for uterine disease. Reproduction 136 361-366.

Elliot, L, KJ McMahon, HT Gier, and GB Marion 1968 Uterus of the cow after parturition: bacterial content. American Journal of Veterinary Research 29 77-81.

Elovitz, MA, Z Wang, EK Chien, DF Rychlik, and M Phillippe 2003 A new model for inflammation-induced preterm birth: the role of platelet-activating factor and Toll-like receptor-4. American Journal of Pathology 163 2103-2111.

Ferrandon, D, JL Imler, C Hetru, and JA Hoffmann 2007 The Drosophila systemic immune response: sensing 
and signalling during bacterial and fungal infections. Nature Reviews. Immunology 7 862-874.

Fischer, C, M Drillich, S Odau, W Heuwieser, R Einspanier, and C Gabler 2010 Selected pro-inflammatory factor transcripts in bovine endometrial epithelial cells are regulated during the oestrous cycle and elevated in case of subclinical or clinical endometritis. Reproduction Fertility and Development 22 818-829.

Garlanda, C, CA Dinarello, and A Mantovani 2013 The Interleukin-1 Family: Back to the Future. Immunity 39 1003-1018.

Gasque, P 2004 Complement: a unique innate immune sensor for danger signals. Molecular Immunology $\mathbf{4 1}$ 1089-1098.

Goubau, D, S Deddouche, and ESC Reis 2013 Cytosolic sensing of viruses. Immunity 38 855-869.

Griffin, JFT, PJ Hartigan, and WR Nunn 1974 Non-specific uterine infection and bovine fertility. I. Infection patterns and endometritis during the first seven weeks post-partum. Theriogenology 1 91-106.

Harris, CL, M Mizuno, and BP Morgan 2006 Complement and complement regulators in the male reproductive system. Molecular Immunology 43 57-67.

Herath, S, DP Fischer, D Werling, EJ Williams, ST Lilly, H Dobson, CE Bryant, and IM Sheldon 2006 Expression and function of Toll-like receptor 4 in the endometrial cells of the uterus. Endocrinology 147 562-570.

Herath, S, ST Lilly, DP Fischer, EJ Williams, H Dobson, CE Bryant, and IM Sheldon 2009a Bacterial lipopolysaccharide induces an endocrine switch from prostaglandin $F_{2 a}$ to prostaglandin $E_{2}$ in bovine endometrium. Endocrinology 150 1912-1920.

Herath, S, ST Lilly, NR Santos, RO Gilbert, L Goetze, CE Bryant, JO White, J Cronin, and IM Sheldon 2009b Expression of genes associated with immunity in the endometrium of cattle with disparate postpartum uterine disease and fertility. Reproductive Biology and Endocrinology 755.

Herath, S, EJ Williams, ST Lilly, RO Gilbert, H Dobson, CE Bryant, and IM Sheldon 2007 Ovarian follicular cells have innate immune capabilities that modulate their endocrine function. Reproduction 134 683-693.

Hirata, T, Y Osuga, Y Hirota, K Koga, O Yoshino, M Harada, C Morimoto, T Yano, O Nishii, O Tsutsumi, and Y Taketani 2005 Evidence for the presence of toll-like receptor 4 system in the human endometrium. Journal of Clinical Endocrinology and Metabolism 90 548-556.

Horne, AW, SJ Stock, and AE King 2008 Innate immunity and disorders of the female reproductive tract. Reproduction 135 739-749.

Irvine, KL, LJ Hopkins, M Gangloff, and CE Bryant 2013 The molecular basis for recognition of bacterial ligands at equine TLR2, TLR1 and TLR6. Veterinary Research 44 50,51-11.

Jacca, S, V Franceschi, A Colagiorgi, M Sheldon, and G Donofrio 2013 Bovine endometrial stromal cells support tumor necrosis factor alpha-induced bovine herpesvirus type 4 enhanced replication. Biology of Reproduction 88135.
Karaghiosoff, M, R Steinborn, P Kovarik, G Kriegshauser, M Baccarini, B Donabauer, U Reichart, T Kolbe, C Bogdan, T Leanderson, D Levy, $T$ Decker, and M Muller 2003 Central role for type I interferons and Tyk2 in lipopolysaccharide-induced endotoxin shock. Nature Immunology 4 471-477.

Lavon, Y, G Leitner, E Klipper, U Moallem, R Meidan, and D Wolfenson 2011 Subclinical, chronic intramammary infection lowers steroid concentrations and gene expression in bovine preovulatory follicles. Domestic Animal Endocrinology 40 98-109.

Lemaitre, B, E Nicolas, L Michaut, JM Reichhart, and JA Hoffmann 1996 The dorsoventral regulatory gene cassette spatzle/Toll/cactus controls the potent antifungal response in Drosophila adults. Cell 86 973-983.

Li, SH, HL Huang, and YH Chen 2002 Ovarian steroidregulated synthesis and secretion of complement C3 and factor B in mouse endometrium during the natural estrous cycle and pregnancy period. Biology of Reproduction 66 322-332.

Machado, VS, G Oikonomou, ML Bicalho, WA Knauer, R Gilbert, and RC Bicalho 2012 Investigation of postpartum dairy cows uterine microbial diversity using metagenomic pyrosequencing of the 16S rRNA gene. Veterinary Microbiology.

Moresco, EM, D LaVine, and B Beutler 2011 Toll-like receptors. Current Biology 21 R488-493.

Poltorak, A, X He, I Smirnova, MY Liu, CV Huffel, X Du, D Birdwell, E Alejos, M Silva, C Galanos, M Freudenberg, P Ricciardi-Castagnoli, B Layton, and B Beutler 1998 Defective LPS signaling in $\mathrm{C} 3 \mathrm{H} / \mathrm{HeJ}$ and $\mathrm{C} 57 \mathrm{BL} / 10 \mathrm{ScCr}$ mice: mutations in Tlr4 gene. Science 282 2085-2088.

Price, JC, JJ Bromfield, and IM Sheldon 2013 Pathogenassociated molecular patterns initiate inflammation and perturb the endocrine function of bovine granulosa cells from ovarian dominant follicles via TLR2 and TLR4 pathways. Endocrinology 154 3377-3386.

Ravel, J, P Gajer, Z Abdo, GM Schneider, SSK Koenig, SL McCulle, S Karlebach, R Gorle, J Russell, CO Tacket, RM Brotman, CC Davis, K Ault, L Peralta, and LJ Forney 2011 Vaginal microbiome of reproductive-age women. Proceedings of the National Academy of Sciences of the United States of America 108 Suppl. 1 4680-4687.

Rice, VM, SD Limback, KF Roby, and PF Terranova 1998 Differential responses of granulosa cells from small and large follicles to follicle stimulating hormone (FSH) during the menstrual cycle and acyclicity: effects of tumour necrosis factor-alpha. Human Reproduction 13 1285-1291.

Richards, JS, Z Liu, and M Shimada 2008 Immune-like mechanisms in ovulation. Trends in Endocrinology and Metabolism 19 191-196.

Roby, KF, and PF Terranova 1990 Effects of tumor necrosis factor-alpha in vitro on steroidogenesis of healthy and atretic follicles of the rat: theca as a target. Endocrinology 126 2711-2718.

Rowson, LE, GE Lamming, and RM Fry 1953 Influence of ovarian hormones on uterine infection. Nature $\mathbf{1 7 1}$ 749-750. 
Sauter, KS, M Brcic, M Franchini, and TW Jungi 2007 Stable transduction of bovine TLR4 and bovine MD-2 into LPS-nonresponsive cells and soluble CD14 promote the ability to respond to LPS. Veterinary Immunology and Immunopathology 118 92-104.

Schroder, K, and J Tschopp 2010 The inflammasomes. Cell 140 821-832.

Schweizer, M, and E Peterhans 2001 Noncytopathic bovine viral diarrhea virus inhibits double-stranded RNA-induced apoptosis and interferon synthesis. Journal of Virology 75 4692-4698.

Seabury, CM, PM Seabury, JE Decker, RD Schnabel, JF Taylor, and JE Womack 2010 Diversity and evolution of 11 innate immune genes in Bos taurus taurus and Bos taurus indicus cattle. Proceedings of the National Academy of Sciences of the United States of America 107 151-156.

Sheldon, IM, and JJ Bromfield 2011 Innate immunity in the human endometrium and ovary. American Journal of Reproductive Immunology 66 Suppl 1 63-71.

Sheldon, IM, M Bushnell, J Montgomery, and AN Rycroft 2004a Minimum inhibitory concentrations of some antimicrobial drugs against bacteria causing uterine infections in cattle. Veterinary Record 155 383-387.

Sheldon, IM, J Cronin, L Goetze, G Donofrio, and HJ Schuberth 2009 Defining postpartum uterine disease and the mechanisms of infection and immunity in the female reproductive tract in cattle. Biology of Reproduction $\mathbf{8 1}$ 1025-1032.

Sheldon, IM, DE Noakes, M Bayliss, and H Dobson 2003a The effect of oestradiol on postpartum uterine involution in sheep. Animal Reproduction Science 78 57-70.

Sheldon, IM, DE Noakes, AN Rycroft, and H Dobson 2003b The effect of intrauterine administration of estradiol on postpartum uterine involution in cattle. Theriogenology 59 1357-1371.

Sheldon, IM, DE Noakes, AN Rycroft, DU Pfeiffer, and H Dobson 2002 Influence of uterine bacterial contamination after parturition on ovarian dominant follicle selection and follicle growth and function in cattle. Reproduction 123 837-845.

Sheldon, IM, and MH Roberts 2010 Toll-like receptor 4 mediates the response of epithelial and stromal cells to lipopolysaccharide in the endometrium. PLOS ONE 5 e12906.

Sheldon, IM, AN Rycroft, B Dogan, M Craven, JJ Bromfield, A Chandler, M Roberts, H., SB Price, RO Gilbert, and KW Simpson 2010 Specific strains of Escherichia coli are pathogenic for the endometrium of cattle and cause pelvic inflammatory disease in cattle and mice. PLOS ONE 5 e9192.
Sheldon, IM, AN Rycroft, and C Zhou 2004b Association between postpartum pyrexia and uterine bacterial infection in dairy cattle. Veterinary Record 154 289-293.

Shimada, M, I Hernandez-Gonzalez, I Gonzalez-Robanya, and JS Richards 2006 Induced expression of pattern recognition receptors in cumulus oocyte complexes: novel evidence for innate immune-like functions during ovulation. Molecular Endocrinology 20 3228-3239.

Spicer, LJ 1998 Tumor necrosis factor-a (TNF-a) inhibits steroidogenesis of bovine ovarian granulosa and thecal cells in vitro. Involvement of TNF-a receptors. Endocrine 8 109-115.

Takeuchi, O, and S Akira 2010 Pattern recognition receptors and inflammation. Cell 140 805-820.

Turner, ML, JC Cronin, GD Healey, and IM Sheldon 2014 Epithelial and stromal cells of bovine endometrium have roles in innate immunity and initiate inflammatory responses to bacterial lipopeptides in vitro via Toll-like receptors TLR2, TLR1 and TLR6. Endocrinology 155 1453-1465.

Tweten, RK 2005 Cholesterol-dependent cytolysins, a family of versatile pore-forming toxins. Infection and Immunity 73 6199-6209.

Wathes, DC, Z Cheng, W Chowdhury, MA Fenwick, R Fitzpatrick, DG Morris, J Patton, and JJ Murphy 2009 Negative energy balance alters global gene expression and immune responses in the uterus of postpartum dairy cows. Physiolical Genomics 39 1-13.

Werling, D, OC Jann, V Offord, EJ Glass, and TJ Coffey 2009 Variation matters: TLR structure and speciesspecific pathogen recognition. Trends in Immunology 30 124-130.

Williams, EJ, DP Fischer, DE Noakes, GC England, A Rycroft, H Dobson, and IM Sheldon 2007 The relationship between uterine pathogen growth density and ovarian function in the postpartum dairy cow. Theriogenology 68 549-559.

Williams, EJ, and IM Sheldon 2003 Clinical evaluation of postpartum vaginal mucus reflects uterine bacterial infection. Reproduction Abstract Series 3091.

Wira, CR, and JV Fahey 2004 The innate immune system: gatekeeper to the female reproductive tract. Immunology 111 13-15.

Woods, DC, JS Schorey, and AL Johnson 2009 Toll-like receptor signaling in hen ovarian granulosa cells is dependent on stage of follicle maturation. Reproduction 137 987-996.

Yan, K, W Zhu, L Yu, N Li, X Zhang, P Liu, Q Chen, Y Chen, and D Han 2013 Toll-like receptor 3 and RIG-I-like receptor activation induces innate antiviral responses in mouse ovarian granulosa cells. Molecular and Cellular Endocrinology 372 73-85. 